\title{
Biological Activities of Two Major Copaiba Diterpenoids and Their Semi-synthetic Derivatives
}

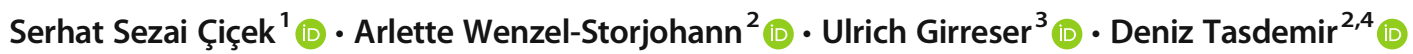

Published online: 21 February 2020

(C) The Author(s) 2020

\begin{abstract}
The oleoresin of Copaifera reticulata Ducke, Fabaceae, is a traditional Brazilian remedy used for a wide range of applications. Commonly named copaiba, the oleoresin has been found to exhibit strong antimicrobial effects in our previous study, which could be attributed to some of its diterpenoid constituents. In order to find new biological activities and to eventually enhance the before observed effects, (-)-polyalthic acid (1) and kaurenoic acid (2), together with eight prepared semi-synthetic derivatives (1a-1c and 2a-2e) were evaluated for their cytotoxic, antibacterial and antifungal properties. Regarding the gram-positive bacteria Enterococcus faecium and methicillin-resistant Staphylococcus aureus, we found that both the exocylic methylene group and the carboxyl group were crucial for the activity against these two clinically relevant bacterial strains. Investigation of the antifungal activity, in contrast, showed that the carboxyl group is unnecessary for the effect against the dermatophytes Trichophyton rubrum and Cryptococcus neoformans, indicated by low micromolar $\mathrm{IC}_{50}$ values for both (-)-polyalthic acid diethylamide (1a) as well as (-)-polyalthic acid methyl ester (1) $)$. Apart from studying the biological activity, the structure of one semi-synthetic derivative, compound $\mathbf{1 c}$, is being reported for the first time. During the course of the structure elucidation of the new compound, we discovered inconsistencies regarding the stereochemistry of polyalthic acid and its stereoisomers, which we clarified in the present work.
\end{abstract}

Keywords Natural product $\cdot$ Partial synthesis $\cdot$ Traditional medicine $\cdot$ Diterpene acid $\cdot$ MRSA $\cdot$ NMR

\section{Introduction}

Copaiba, or copaiba oil, is the name of an oleoresin obtained from selected species of the genus Copaifera, Fabaceae (Leandro et al. 2012). Copaiba is widely used in the traditional

Electronic supplementary material The online version of this article (https://doi.org/10.1007/s43450-020-00002-y) contains supplementary material, which is available to authorized users.

Serhat Sezai Çiçek

scicek@pharmazie.uni-kiel.de

1 Pharmazeutisches Institut, Abteilung Pharmazeutische Biologie, Christian-Albrechts-Universität zu Kiel, Kiel, Germany

2 GEOMAR Centre for Marine Biotechnology, Research Unit Marine Natural Products Chemistry, GEOMAR Helmholtz Centre for Ocean Research Kiel, Kiel, Germany

3 Pharmazeutisches Institut, Abteilung Pharmazeutische und Medizinische Chemie, Christian-Albrechts-Universität zu Kiel, Kiel, Germany

4 Christian-Albrechts-Universität zu Kiel, Kiel, Germany medicine in Brazil and other Latin American countries for treatment of various diseases, such as skin and urinary tract infections, respiratory diseases, ulcers, rheumatism, herpes, tumours and tetanus disease (Ohsaki et al. 1994). Due to its antibacterial, antihelminthic, trypanocidal and leishmanicidal applications, the oleoresin represents an important natural remedy for people without access to modern medicine and commercial drugs (Ohsaki et al. 1994; Leandro et al. 2012).

Copaiba consists of sesquiterpenes and diterpenes, the latter usually being present as diterpene acids (Leandro et al. 2012). Sesquiterpenes represent the main compounds that can count up to $98 \%$ of the oleoresin by weight, and show a rather stable metabolite pattern, with $\beta$-caryophyllene being the major constituent in most Copaifera species (Leandro et al. 2012). However, $\beta$ bisabolene has been identified as the major sesquiterpene of Copaifera reticulata Ducke collected in the Pará region (northern Brazil) (Pfeifer Barbosa et al. 2019). In contrast to the sesquiterpenes, copaiba diterpenoids show much higher interspecific variation and therefore attracted particular interest in recent years due to varying biological 
activities observed for the different Copaifera species (Leandro et al. 2012). These activities comprise cytotoxic, antibacterial, antifungal and antiprotozoal properties as part of numerous in vitro and in vivo studies attempting to attribute the detected effect to certain compounds or compound classes.

The cytotoxic potential of the copaiba oleoresin seems to mainly derive from its sesquiterpene constituents, as respective investigations on diterpenoids resulted in weak to moderate (but then non-selective) cytotoxic effects (Cavalcanti et al. 2009; Pfeifer Barbosa et al. 2019). The only exception is kolavenol, which showed antitumour effects against IMC carcinoma in mice, determined by an increase of life span of the animals (Ohsaki et al. 1994). In contrast, the main sesquiterpenes showed selective moderate ( $\beta$-bisabolol) to pronounced ( $\beta$-caryophyllene and $\beta$-caryophyllene oxide) cytotoxic activities (Kubo et al. 1996; Yeo et al. 2016). With respect to their higher concentration in the oleoresin, it is more likely that sesquiterpenes are responsible for the antitumour properties of copaiba (Pfeifer Barbosa et al. 2019).

On the other hand, copaiba diterpenoids showed good in vitro activities against causative agents of infectious parasitic diseases. Copalic acid, $3 \beta$-hydroxycopalic acid and methyl copalate, for example, inhibited the growth of the amastigote forms of Trypanosoma cruzi, whereas kaurenoic acid (2) and some of its semi-synthetic derivatives were found active against its erythrocytic trypomastigote forms (Vieira et al. 2002; Izumi et al. 2012). (-)-Polyalthic acid (1), another major copaiba diterpenoid showed activity against $T$. brucei and amastigote forms of Leishmania donovani (Mizuno et al. 2015). Also here, semi-synthetic derivatives were prepared, of which some showed comparable to slightly higher activities than the natural product. However, neither (-)-polyalthic acid (1) nor any of its derivatives was leishmanicidal against promastigote forms of $L$. donovani.

Moreover, copaiba diterpenoids revealed antibacterial activity against both gram-positive and gram-negative bacteria, with pronounced effects against Bacillus subtilis and five different Streptococcus species (Tincusi et al. 2002; Souza et al. 2011). In our previous study, we reported strong inhibitory effects against the two clinically relevant bacterial strains methicillin-resistant Staphylococcus aureus (MRSA) and Enterococcus faecium for three diterpene acids, of which one was kaurenoic acid (2) (Pfeifer Barbosa et al. 2019). In the same study, antidermatophytic activity against two Trichopyhton species was detected for (-)-polyalthic acid (1), the major diterpenoid in the Copaifera reticulata oleoresin, along with weak [(-)-polyalthic acid, 1] to moderate (kaurenoic acid, 2) cytotoxic effects against six cancer cell lines. These findings prompted us to prepare semi-synthetic derivatives of both compounds in an attempt to enhance their antimicrobial and cytotoxic properties and to get more insights into their structure-activity-relationships (SAR). Furthermore, additional screenings were performed to discover new lead compounds against both human and plant pathogens.

\section{Materials and Methods}

\section{Plant Material and Chemicals}

(-)-Polyalthic acid (1) and kaurenoic acid (2) were isolated from the oleoresin of Copaifera reticulata Ducke, Fabaceae, as described in our previous studies (Çiçek et al. 2018; Pfeifer Barbosa et al. 2019). $N$-Chlorosuccinimide (98\%) and triphenylphosphine (ReagentPlus, 99\%), sulphuric acid (puriss., analytical grade) and LC-MS grade formic acid were purchased from Sigma-Aldrich Co., St. Louis, MO, USA. Hydrochloric acid (25\%, analytical grade) was obtained from Honeywell, Seelze, Germany, while sodium hydroxide solution $(2 \mathrm{~N})$ was purchased from Carl Roth $\mathrm{GmbH}$, Karlsruhe, Germany. Diethylamine (for synthesis), acetone (analytical grade), methanol (gradient grade or LC-MS grade) and water (LC-MS grade) as well as other analytical grade solvents used for purification were obtained from VWR International $\mathrm{GmbH}$, Darmstadt, Germany. Solid phase extraction (SPE) columns (Chromabond SB $3 \mathrm{ml} / 500 \mathrm{mg}$ ) were obtained from Macherey-Nagel GmbH \& Co. KG, Düren, Germany. Deuterated methanol (Lot P3021, 99.80\%), deuterated DMSO (Lot S1051, 99.80\%) and deuterated chloroform (Lot Q1981, 99.80\%) for NMR spectroscopy were obtained from Eurisotop GmbH, Saarbrücken, Germany. Conventional 5-mm sample tubes were purchased from Rototec-Spintec $\mathrm{GmbH}$, Griesheim, Germany.

\section{General Experimental Procedures}

Semi-synthetic derivatives of compounds 1 and $\mathbf{2}$ were prepared in 5-ml V-Vials (Wheaton, Millville, NJ, USA) using a RET basic magnetic stirrer with integrated heater (IKA-Werke GmbH \& Co. KG, Staufen, Germany). LCDAD-ELSD analysis was accomplished as stated in Çiçek et al. (2018); LC-MS and GC-MS analyses were conducted as described in Pfeifer Barbosa et al. (2019). Highresolution MS spectrum was recorded on micrOTOF IIHigh-performance TOF-MS system (Bruker®, Billerica, MA, USA) equipped with an electrospray ionisation source. Specific rotation of the compounds measured in methanol on a Jasco P-2000 polarimeter (Jasco, Pfungstadt, Germany). NMR spectra were recorded using a Bruker Avance III 400 NMR spectrometer operating at $400 \mathrm{MHz}$ for the proton channel and $100 \mathrm{MHz}$ for the ${ }^{13} \mathrm{C}$ 
channel with a $5 \mathrm{~mm}$ PABBO broad band probe with a $\mathrm{z}$ gradient unit at $293 \mathrm{~K}$ (Bruker BioSpin GmbH, Rheinstetten, Germany). Reference values were 3.31 $\left({ }^{1} \mathrm{H}\right)$ and $49.15 \mathrm{ppm}\left({ }^{13} \mathrm{C}\right)$ for methanol as well as 2.50 $\left({ }^{1} \mathrm{H}\right)$ and $39.51 \mathrm{ppm}\left({ }^{13} \mathrm{C}\right)$ for DMSO, respectively.

\section{Preparation of Derivatives}

\section{Carboxyl Amides}

The compound $(100 \mu \mathrm{mol})$ and triphenylphosphine were dissolved in $2 \mathrm{ml}$ of dichloromethane, and the mixture was cooled in an ice bath. After adding $110 \mu \mathrm{mol}$ of $N$-chlorosuccinimide in small portions, the mixture was vigorously stirred for $30 \mathrm{~min}$ at room temperature. Diethylamine $(110 \mu \mathrm{mol})$ in $100 \mu \mathrm{mol}$ of pyridine was added, and the mixture was stirred for another $10 \mathrm{~min}$ at room temperature. The mixture was subsequently concentrated under reduced pressure, and the by-products were removed by filtration and solid phase extraction using hexane as solvent to afford $6.2 \mathrm{mg}$ of (-)-polyalthic acid- $N, N$-diethylamide (1a) and $7.5 \mathrm{mg}$ kaurenoic acid- $N, N$-diethylamide (2a).

\section{Carboxyl Esters}

The formation of ester was accomplished in the same manner except that diethylamine was substituted with methanol in the last reaction step. This reaction yielded $4.2 \mathrm{mg}(-)$-polyalthic acid methyl ester (1b) and $7.3 \mathrm{mg}$ kaurenoic acid methyl ester (2b).

\section{Dihydroxy Derivatives}

The compound $(100 \mu \mathrm{mol})$ was dissolved under heating in a mixture of $800 \mu \mathrm{l}$ of water and $200 \mu \mathrm{l}$ of $2 \mathrm{M}$ sodium hydroxide. The solution was subsequently cooled to $5{ }^{\circ} \mathrm{C}$, and $250 \mathrm{mg}$ of ice was added before adding a cold solution of $20.5 \mathrm{mg}$ potassium permanganate in $500 \mu \mathrm{l}$ of water over a period of $30 \mathrm{~min}$. The mixture was kept at 0 to $5{ }^{\circ} \mathrm{C}$ for another $60 \mathrm{~min}$ before the precipitate was filtered off and washed with hot water. The filtrate was acidified with acetic acid, and the resulting precipitate was filtered of and washed with cold water and dried to afford $8.8 \mathrm{mg}$ of $(4 S, 8 S)$-15,16-epoxy-8,17-dihydroxy-13(16),14-entlabdadien-18-oic acid [ $(1 S, 4 a R, 5 S, 6 S, 8 a S)$-5-[2-(furan-3yl)ethyl]-1,4a-dimethyl-6-hydroxy-6-hydroxymethyl3,4,5,7,8,8a-hexahydro-2H-naphthalene-1-carboxylic acid] (1c) and 13.0 mg of 16 $\alpha$,17-dihydroxy-ent-kauran-19-oic acid (2c), respectively, after recrystallisation from a mixture of hexane and tert-butyl methyl ether.

\section{Monohydroxy Derivative}

Compound $2(50 \mathrm{mg})$ was dissolved in a mixture of $1500 \mu \mathrm{l}$ acetone, $350 \mu \mathrm{l}$ water and $150 \mu \mathrm{l}$ hydrochloric acid (25\%) and kept at $56^{\circ} \mathrm{C}$ for $2 \mathrm{~h}$ under stirring. The solution was then transferred to a beaker containing $10 \mathrm{ml}$ of water , and the resulting precipitate was filtered and recrystallised from acetone to obtain $12.2 \mathrm{mg}$ of $16 \alpha$-hydroxy-ent-kauran-19-oic acid (2d).

\section{Methoxy Derivative}

Compound $2(50 \mathrm{mg})$ was dissolved in a mixture of $2500 \mu \mathrm{l}$ methanol and $150 \mu \mathrm{l}$ sulphuric acid and kept at $65{ }^{\circ} \mathrm{C}$ for $3 \mathrm{~h}$ under stirring. The solution was subsequently cooled to $-18{ }^{\circ} \mathrm{C}$ and the resulting precipitate was filtered and recrystallised from methanol to obtain $7.9 \mathrm{mg}$ of $16 \alpha$-methoxy-ent-kauran-19-oic acid (2e).

\section{Biological Activity Testing}

\section{Antibacterial and Antiyeast Activity}

The samples were tested against the ESKAPE panel of multidrug resistant bacterial human pathogens, including the grampositive bacteria Enterococcus faecium (DSM 20477) and methillicin-resistant Staphylococcus aureus (MRSA, DSM 18827), and the gram-negative bacteria Klebsiella pneumoniae (DSM 30104), Acinetobacter baumannii (DSM 30007), Pseudomonas aeruginosa (DSM 1128) and Escherichia coli (DSM 1576). Furthermore the activity of the samples against four phytopathogenic bacteria, Pseudomonas syringae (DSM 50252), Xanthomonas campestris (DSM 2405), Erwinia amylovora (DSM 50901) and Ralstonia solanacearum (DSM 9544), and against two human pathogen yeasts, Candida albicans (DSM 1386) and Cryptococcus neoformans (DSM 6973), was carried out. All test strains were purchased from Leibniz Institute DSMZGerman Collection of Microorganisms and Cell Cultures, Braunschweig, Germany.

The bacteria were cultivated in TSB medium (1.2\% tryptic soy broth, $0.5 \% \mathrm{NaCl}$ ), except $E$. faecium which was cultivated in M92 medium (3\% trypticase soy broth, $0.3 \%$ yeast extract, $\mathrm{pH}$ 7.0-7.2) and $R$. solanacearum which was grown in M186 (1\% glucose, $0.5 \%$ peptone from soymeal, $0.3 \%$ malt extract, 0.3 yeast extract). The cultivation of C. albicans took place in M186/3 (0.3\% glucose, $0.17 \%$ peptone from soymeal, $0.1 \%$ malt extract, $0.1 \%$ yeast extract) and for C. neoformans M186 was used as well. Overnight cultures of the test organisms were prepared and diluted to an optical density $(600 \mathrm{~nm})$ of $0.01-0.03$. To prepare the assay, the test samples $(20 \mathrm{mg} / \mathrm{ml}$ DMSO stock solution) were dissolved in medium and transferred into a 96-well microtiter plate and $200 \mu \mathrm{l}$ of the cell suspension cultures was added to each well. The final assay concentration of the substances was $100 \mu \mathrm{g} / \mathrm{ml}$. The inoculated microplates were incubated for $5 \mathrm{~h}$ at $37{ }^{\circ} \mathrm{C}$ and $200 \mathrm{rpm}$ 
(E. faecium without shaking), or $28^{\circ} \mathrm{C}$ and $200 \mathrm{rpm}$ for $7 \mathrm{~h}$ for the phytopathogen bacteria and $C$. neoformans, respectively. To detect the inhibitory effect of the substances $10 \mu \mathrm{l}$ of a resazurin solution $(0.3 \mathrm{mg} / \mathrm{ml}$ phosphate-buffered saline) was added to the microplates and incubated again for 5-30 min before the fluorescence signal (560 nm/590 nm) was measured using the microplate reader (Tecan Infinite M200). For E. faecium the $\mathrm{pH}$ indicator bromocresol purple was used to determine the acidification caused by growing, and for $R$. solanacearum and $C$. neoformans the optical density at $600 \mathrm{~nm}$ after incubation time was recorded using the microplate reader as well. The resulting values were compared with a positive control (chloramphenicol for the bacteria, except $R$. solanacearum where tetracycline was used, nystatin for $C$. albicans and amphotericin B for C. neoformans) and a negative control (no compound) on the same plate. For $\mathrm{IC}_{50}$ determination, a dilution series was prepared and the $\mathrm{IC}_{50}$ value was calculated by Excel or GraphPad Prism as the concentration that shows 50\% inhibition of viability on the basis of a negative control (no compound).

\section{Cytotoxic Activity and Activity Against Dermatophytic Fungi}

Measurements of cytotoxic activity and activity against dermatophytic fungi were performed as described in our previous study (Pfeifer Barbosa et al. 2019).

\section{Results and Discussion}

\section{Preparation of Semi-synthetic Derivatives 1a-1c and 2a-2e}

Semi-synthetic derivatives of (-)-polyalthic acid (1) and kaurenoic acid (2) were prepared on a small scale using 5-ml $\mathrm{V}$-Vials and applying between 30 and $50 \mathrm{mg}$ of diterpene acid. Derivatisations included the carboxyl group and the exocyclic methylene group, respectively. Thus, $N, N$-diethyl amide (1a and $\mathbf{2 a}$ ) and methyl ester (1) and $\mathbf{2 b}$ ) as well as dihydroxy derivatives (1c and 2c) of both parent molecules were prepared as described in the Experimental section. Additionally, one monohydroxy derivative (2d) and one methoxy derivative (2e) of kaurenoic acid were prepared. All products were analysed by TLC using different mixtures of dichloromethane and ethanol as solvent and vanillin-sulphuric acid as spray reagent. Amides and esters were additionally checked by $\mathrm{GC}^{-\mathrm{MS}^{2}}$, whereas the other derivatives were analysed by UHPLC-DAD/ELSD and UHPLC-MS ${ }^{2}$. For structure elucidation, one- and two-dimensional NMR experiments were performed.

\section{Amides and Methyl Esters}

Amidation and esterification of diterpenoids was accomplished using $N$-chlorosuccinimide and triphenylphosphine for conversion of the carboxylic acids and adding either $N, N$-diethylamine or methanol in pyridine to the reaction mixture as described in the protocols of Frøyen (1994, 1995). The experiments were therefore downsized to $100 \mu \mathrm{mol}$ of reactants (instead of $5 \mathrm{mmol}$ ) except for dichloromethane, of which $2 \mathrm{ml}$ (instead of $6 \mathrm{ml}$ ) was used. Purification was conducted as described in the protocols (concentration, filtration and washing with diethyl ether, silica gel column with ether as eluent). However, for the last purification step another silica gel column with $n$-hexane as eluent was preferred over crystallisation or distillation, respectively. All four derivatives were subsequently analysed by GC-MS ${ }^{2}$ showing the expected $\mathrm{m} / \mathrm{z}$ ratios of $371(\mathbf{1 a}), 357(\mathbf{2 a}), 330(\mathbf{1 b})$, and $316(\mathbf{2 b})$ and by NMR spectroscopy comparing their spectra to literature data (Narayanan and Venkatasubramanian 1965; Vieira et al. 2002; Mizuno et al. 2015; Santos et al. 2016). The methyl esters (1b and $\mathbf{2 b}$ ) were additionally identified by comparing their mass spectra to the spectra available in the NIST database.

\section{Dihydroxy Derivatives}

Dihydroxy derivatives of compounds $\mathbf{1}$ and $\mathbf{2}$ were prepared with potassium permanganate in alkaline medium following a protocol for the hydroxylation of lambertianic acid (Chernov et al. 2006). Lambertianic acid is an epimer of (+)-polyalthic acid at position C-4 and thus a stereoisomer of (-)-polyalthic acid (1), showing different configurations at positions C-5, $\mathrm{C}-9$, and C-10. Using scales of $100 \mu \mathrm{mol}$ of diterpenoid instead of $20 \mathrm{mmol}$, the reaction yielded $(4 S, 8 S)$-15, 16-epoxy8,17-dihydroxy-13(16),14-ent-labdadien-18-oic acid (1c), a previously undescribed compound, and the known compound 16 $\alpha$,17-dihydroxy-ent-kauran-19-oic acid (2c). Whereas the latter compound was identified by comparison of MS and NMR spectra to literature data (Song et al. 2018), structure elucidation of compound 1c is described in "Structure Elucidation and Configuration of Compound 1 $\mathbf{c}$ ".

\section{Monohydroxy and Methoxy Derivatives}

Preparation of $16 \alpha$-hydroxy-ent-kauran-19-oic acid (2d), $16 \alpha$-methoxy-ent-kauran-19-oic acid (2e) was performed using acid-catalysed hydroxylation and methoxylation, respectively (Cavalcanti et al. 2009). For these reactions, amounts of $50 \mathrm{mg}$ were applied instead of $1 \mathrm{~g}$ and the resulting products were identified by comparison of their MS and NMR data to literature (Chen et al. 2000; Yaouba et al. 2018). 


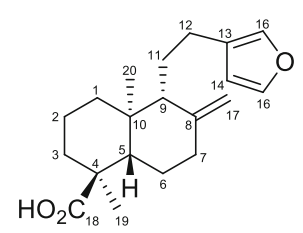

1

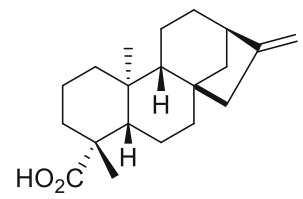

2

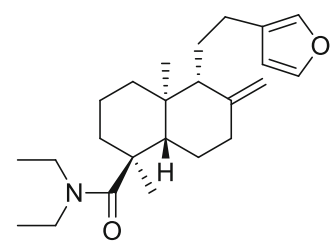

$1 \mathbf{a}$

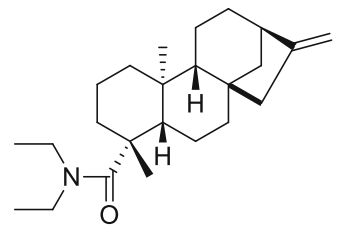

$2 a$

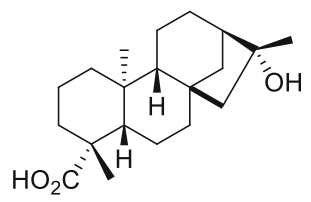

2d<smiles>CO[C@H]1CCC[C@H]2CCc3cocc3CC[C@H]21</smiles>

$1 b$

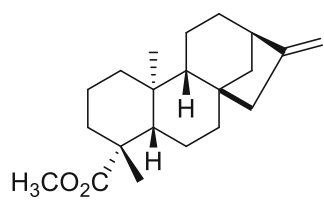

$2 b$

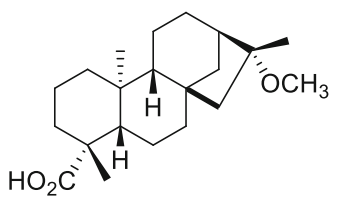

2d

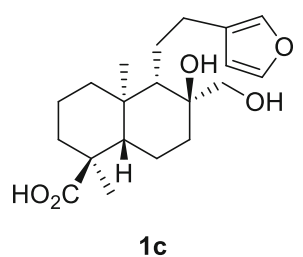

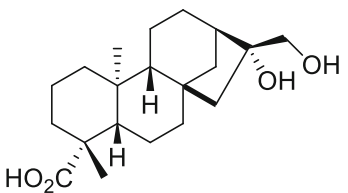

2c

\section{Structure Elucidation and Configuration of Compound 1c}

As mentioned above, compound 1c was synthesised using a protocol (Chernov et al. 2006) reported for the transformation of lambertianic acid, a stereoisomer of (-)-polyalthic acid (1). The side chain at C-10 of lambertianic acid is $\beta$ oriented leading to $\alpha$-oriented hydroxylation at position C-8, due to steric hindrance by the furanoethyl group. In contrast, (-)-polyalthic acid (1) shows an $\alpha$-oriented side chain at $\mathrm{C}-10$; hence, the resulting hydroxy group at position C- 8 had to have $\beta$-orientation and the hydroxymethylene group $\alpha$-orientation instead. The $\alpha$-orientation of the hydroxymethylene group was confirmed using NOESY, where NOE correlations were observed for the protons at $\mathrm{C}-17$ on both the methylene group at $\mathrm{C}-11$ and the $\mathrm{C}-20$ methyl group. Thus, the structure was identified as $(4 S, 8 S)$ 15,16-epoxy-8,17-dihydroxy-13(16),14-ent-labdadien-18oic acid or (rel-1S,4aR,5S,6S,8aS)-5-[2-(furan-3-yl)ethyl]1,4a-dimethyl-6-hydroxy-6-hydroxymethyl-3,4,5,7,8,8ahexahydro- $2 H$-naphthalene-1-carboxylic acid, respectively<smiles>C[C@]12CCC[C@@H](C(=O)O)[C@@H]1CCC1=CC=CC1CCc1cocc12</smiles>

3

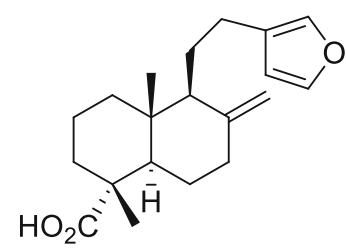

4

(see "Stereochemistry of Polyalthic Acid, Daniellic Acid and Lambertianic Acid"). Same as (-)-polyalthic acid (1), the new compound (1c) showed a negative specific rotation value $\left[\alpha_{\mathrm{D}}{ }^{20}-14.9, c=0.1\right.$, methanol). HR-ESI-MS revealed $m / z$ ratios of $373.1994[M+\mathrm{Na}]^{+}$and 723.4090 [2 $\mathrm{M}+\mathrm{Na}]^{+}$, corresponding to the molecular formula $\mathrm{C}_{20} \mathrm{H}_{30} \mathrm{O}_{5}$ with the experimental mass of $M 350.2096$ (calculated mass was $M$ 350.2093). NMR data for compound 1c is given in Table 1.

\section{Stereochemistry of Polyalthic Acid, Daniellic Acid and Lambertianic Acid}

As described above, hydroxylation of (-)-polyalthic acid (1) followed a protocol of Chernov et al. (2006), who used lambertianic acid as starting material. However, during the course of the structure determination of compound 1c and assignment of the absolute configuration, some inconsistencies have been observed with regard to the stereochemistry of polyalthic acid and its structural isomers, which we attempted to resolve hereafter.<smiles>C=C1CC[C@H]2[C@@H](C)[C@@H](C(=O)O)CCC[C@]2(C)[C@H]1CCc1ccoc1</smiles>

5<smiles>C[C@]1(C(=O)O)CCC[C@]2(C)C1CC[C@](O)(CO)[C@H]2CCc1ccoc1</smiles>

6 
Table 1 NMR data for compound $1 \mathbf{c}$ in methanol- $d_{4}$ and DMSO- $d_{6}(400 \mathrm{MHz}, \delta$ in ppm, $J$ in $\mathrm{Hz}$ )

\begin{tabular}{|c|c|c|c|c|}
\hline Position & ${ }^{1} \mathrm{H}$ NMR $\left(\mathrm{CD}_{3} \mathrm{OD}\right)$ & ${ }^{13} \mathrm{C}$ NMR $\left(\mathrm{CD}_{3} \mathrm{OD}\right)$ & ${ }^{1} \mathrm{H}$ NMR (DMSO- $d_{6}$ ) & ${ }^{13} \mathrm{C}$ NMR (DMSO- $\left.d_{6}\right)$ \\
\hline \multirow[t]{2}{*}{1} & $1.77(\mathrm{~m})$ & 41.0 & $1.63(\mathrm{~m})$ & 38.4 \\
\hline & $1.14(\mathrm{~m})$ & & $0.97(\mathrm{~m})$ & \\
\hline \multirow[t]{2}{*}{2} & $1.68(\mathrm{~m})$ & 19.1 & $1.54(\mathrm{~m})$ & 16.8 \\
\hline & $1.59(\mathrm{~m})$ & & $1.46(\mathrm{~m})$ & \\
\hline \multirow[t]{2}{*}{3} & $2.24(\mathrm{~m})$ & 38.7 & $2.06(\mathrm{~m})$ & 36.8 \\
\hline & $1.29(\mathrm{~m})$ & & $1.06(\mathrm{~m})$ & \\
\hline 4 & & 48.6 & & 46.4 \\
\hline 5 & $1.89(\mathrm{~s})$ & 50.3 & $1.68(\mathrm{~s})$ & 49.9 \\
\hline \multirow[t]{2}{*}{6} & $1.47(\mathrm{~m})$ & 24.4 & $1.31(\mathrm{~m})$ & 22.0 \\
\hline & $1.27(\mathrm{~m})$ & & $1.05(\mathrm{~m})$ & \\
\hline \multirow[t]{2}{*}{7} & $1.81(\mathrm{~m})$ & 38.8 & $1.64(\mathrm{~m})$ & 36.1 \\
\hline & $1.58(\mathrm{~m})$ & & $1.45(\mathrm{~m})$ & \\
\hline 8 & & 76.8 & & 74.4 \\
\hline 9 & $1.42(\mathrm{~s})$ & 62.8 & $1.25(\mathrm{~s})$ & 60.6 \\
\hline 10 & & 39.7 & & 37.6 \\
\hline \multirow[t]{2}{*}{11} & $1.87(\mathrm{~m})$ & 27.5 & $1.74(\mathrm{~m})$ & 25.0 \\
\hline & $1.46(\mathrm{~m})$ & & $1.30(\mathrm{~m})$ & \\
\hline \multirow[t]{2}{*}{12} & $2.63(\mathrm{~m})$ & 29.9 & $2.35(\mathrm{~m})$ & 27.5 \\
\hline & $2.48(\mathrm{td}, 12.1,3.9)$ & & $2.06(\mathrm{~m})$ & \\
\hline 13 & & 126.1 & & 125.6 \\
\hline 14 & $6.35(\mathrm{~d}, 0.9)$ & 112.5 & $6.36(\mathrm{~d}, 0.8)$ & 111.3 \\
\hline 15 & $7.39(\mathrm{t}, 1.4)$ & 144.2 & $7.53(\mathrm{t}, 1.5)$ & 142.8 \\
\hline 16 & $7.30(\mathrm{~s})$ & 140.3 & $7.42(\mathrm{~s})$ & 138.6 \\
\hline \multirow[t]{2}{*}{17} & $3.65(\mathrm{~d}, 10.8)$ & 64.1 & $3.38(\mathrm{~d}, 10.7)$ & 61.3 \\
\hline & $3.51(\mathrm{~d}, 7.9)$ & & $3.27(\mathrm{~d}, 10.7)$ & \\
\hline 18 & & n.o. & & 180.6 \\
\hline 19 & $1.16(\mathrm{~s})$ & 17.5 & $1.01(\mathrm{~s})$ & 16.0 \\
\hline 20 & $0.85(\mathrm{~s})$ & 17.3 & $0.72(\mathrm{~s})$ & 15.5 \\
\hline
\end{tabular}

n.o., not observed

The first two of these four compounds to be reported were (-)-polyalthic acid (1) and daniellic acid in 1961. As the absolute configuration has not been determined yet, (-)polyalthic acid is correctly described as (rel- $1 S, 4 a S, 5 R, 8 a S)$ 5-[2-(furan-3-yl)ethyl]-1,4a-dimethyl-6-methylenedecahydronaphthalene-1-carboxylic acid. Daniellic acid was isolated from Daniellia oliveri (Fabaceae, Caesalpinioideae) (Haeuser and Ourisson 1961), whereas (-)-polyalthic acid (1) was obtained from Polyalthia fragrans (Annonaceae) and named polyalthic acid (Gopinath et al. 1961). Both compounds were attributed to possess the "wrong" configuration corresponding to an ent-labdane scaffold and showing negative optical rotation values. Both compounds are epimers, only differing in the orientation of the carboxyl group at position C-4, which is axial for daniellic acid and equatorial for (-)polyalthic acid (1). Five years later, the first report for lambertianic acid, isolated from Pinus lambertiana (Pinaceae), was made (Dauben and Dauben and German 1965). Lambertianic acid was found to be the optical antipode of daniellic acid and thus to be the first furan diterpene with the so-called normal labdane configuration. The last of the four isomers was the optical antipode of (-)-polyalthic acid (1), isolated from Sequoia semperivirens, Taxodiaceae (Ohta and Nawamaki 1978). However, instead of choosing an original trivial name (e.g. semperiviric acid), the authors named the compound (+)-polyalthic acid, being (rel-1R,4aR, $5 S, 8 a R)$ 5-[2-(furan-3-yl)ethyl]-1,4a-dimethyl-6-methylenedecahydronaphthalene-1-carboxylic acid. Thus, from then onwards, two forms of polyalthic acid existed in the literature. Except for the last 3 years, where (+)- and (-)- or ent- prefixes were used to differentiate between the two enantiomers (Bardají et al. 2016; Borges et al. 2016; Carneiro et al. 2018; Çiçek et al. 2018; Pfeifer Barbosa et al. 2019; Senedese et al. 2019), only one publication in almost four decades adopted such prefixes (Miyazawa et al. 1995). The missing stereochemistry did not represent a problem as long as the studies were dealing with Copaifera, because the ent-configuration is abundant in this genus (Leandro et al. 2012). However, some 
studies investigated plant species from other plant families; wherefore, the identity of the right enantiomer remained unknown. Thus, reporting the right configuration for polyalthic acid is absolutely necessary.

Another problem is that the introduction of (+)-polyalthic acid and the recent use of the ent-prefix for differentiation of the enantiomers seems to have caused confusions in some large databases, such as SciFinder ${ }^{\mathrm{TM}}$ or Reaxys ${ }^{\mathrm{TM}}$. For example, when searching for structure templates of polyalthic acid in the Reaxys database, three suggestions are delivered, of which two display (-)-polyalthic acid and the other one shows (+)-polyalthic acid. However, by using the name entpolyalthic acid, also two structures are displayed, one for (-)-polyalthic acid and the second one for daniellic acid. For both compounds their semisystematic names are given along with twelve references per compound, thus giving equal priority to the correct and the incorrect structure. By looking for chemical structures in the SciFinder database, the name polyalthic acid leads to the structure of (-)-polyalthic acid, which is historically correct, whereas the search for entpolyalthic acid gives the structure of daniellic acid and thus the wrong compound. Alas, these inconsistencies or confusions, respectively, led to several publications showing the structure of daniellic acid instead of (-)-polyalthic acid (Leandro et al. 2012, Çiçek et al. 2018, Da Silva et al. 2017; Pfeifer Barbosa et al. 2019). The mentioned problems can be partly dealt with by using the prefixes of the respective optical rotations, leading to the exact structures for both (+)- and (-)polyalthic acid in the Reaxys database and also to the correct structure for (+)-polyalthic acid with the SciFinder database. Only the name (-)-polyalthic acid does not yield any results with SciFinder, but as the name polyalthic acid leads to the structure of (-)-polyalthic acid and the optical rotation can be retrieved from the experimental details, at least no misinformation is spread. We therefore suggest to use the prefix for the optical rotation only.

\section{Cytotoxic Effects}

In our previous study, we investigated the effect of copaiba diterpenoids against A-375, HepG2, HT-29, HCT-116, A-549 and MDA-MB-231 cancer cell lines as well as non-cancerous $\mathrm{HaCaT}$ cell lines resulting in weak to moderate non-selective cytotoxic effects with $\mathrm{IC}_{50}$ values of 66.2 to $96.4 \mu \mathrm{g} / \mathrm{ml}$ for (-)-polyalthic acid (1) and 50.2 to $79.6 \mu \mathrm{g} / \mathrm{ml}$ for kaurenoic acid (2) (Pfeifer Barbosa et al. 2019). Thus, the effect of derivatising either the carboxyl group or the exocyclic methylene group was the main aim of the present study. In a first screening at a concentration of $100 \mu \mathrm{g} / \mathrm{ml}$ no effects for derivatives without the exocyclic methylene group (1c and $\mathbf{2 c}-$ 2e) were observed (Table S1). Compounds $\mathbf{2 d}$ and $\mathbf{2 e}$ were earlier tested on four different cancer cell lines (K562, HL-60, MDA-MB435 and SF295) as well as on healthy peripheral blood mononuclear cells and compared with kaurenoic acid (Cavalcanti et al. 2009). Also here, the cytotoxic effect disappeared after derivatisation; wherefore, the authors hypothesised that the exocyclic methylene group is crucial for the cytotoxic effect.

However, also derivatisation of the carboxyl group (compounds $\mathbf{1 a}, \mathbf{1 b}, \mathbf{2 a}$ and $\mathbf{2 b}$ ) did not result in total growth inhibition at $100 \mu \mathrm{g} / \mathrm{ml}$, but rather affected healthy $\mathrm{HaCaT}$ cells at this concentration. Because of the only low cytotoxic activity of compounds $\mathbf{1}$ and $\mathbf{2}$ found in our previous study and the not too promising results of the preliminary screening, we decided to focus on the antimicrobial effects of our compounds.

\section{Antibacterial Effects}

Due to the strong effects of (-)-polyalthic acid (1) and especially kaurenoic acid (2) against E. faecium and methicillinresistant Staphylococcus aureus (MRSA) (Pfeifer Barbosa et al. 2019), additional assays on Klebsiella pneumoniae, Acinetobacter baumannii, Pseudomonas aeruginosa and Escherichia coli as well as phytopathogens Pseudomonas syringae, Xanthomonas campestris, Erwinia amylovora and Ralstonia solanacearum were included in our investigations (Table S2). However, at a concentration of $100 \mu \mathrm{g} / \mathrm{ml}$, activity of compounds $\mathbf{1}$ and $\mathbf{2}$ as well as their derivatives against the additionally investigated organisms was rather low or nonexistent in contrast to the already known effects against E. faecium and MRSA. But also against the latter two strains derivatisations did not lead to an improved activity (Table 2). Here, only compound $\mathbf{2 c}$ showed (at least moderate) effects, with $\mathrm{IC}_{50}$ values of $59.7 \mu \mathrm{g} / \mathrm{ml}$ against $E$. faecium and $34.7 \mu \mathrm{g} / \mathrm{ml}$ against MRSA. In contrast, (-)-polyalthic acid (1) and kaurenoic acid (2) showed $\mathrm{IC}_{50}$ values of 8.5 and $2.3 \mu \mathrm{g} / \mathrm{ml}$ against E. faecium as well as 8.9 and $3.4 \mu \mathrm{g} / \mathrm{ml}$

Table 2 Antibacterial effects of (-)-polyalthic acid (1), kaurenoic acid (2) and their derivatives. The $\mathrm{IC}_{50}$ values are in $\mu \mathrm{g} / \mathrm{ml}$. Positive controls were ampicillin (Enterococcus faecium) and chloramphenicol (MRSA)

\begin{tabular}{lll}
\hline & E. faecium & MRSA \\
\hline Compound 1 & $8.5 \pm 0.4$ & $8.9 \pm 0.8$ \\
Compound 1a & $>100$ & $>100$ \\
Compound 1b & $>100$ & $>100$ \\
Compound 1c & $>100$ & $>100$ \\
Compound 2 & $2.3 \pm 0.2$ & $3.4 \pm 0.2$ \\
Compound 2a & $>100$ & $>100$ \\
Compound 2b & $>100$ & $>100$ \\
Compound 2c & $59.7 \pm 0.1$ & $34.7 \pm 2.5$ \\
Compound 2d & $>100$ & $>100$ \\
Compound 2e & $>100$ & $>100$ \\
Positive control & $0.4 \pm 0.1$ & $1.2 \pm 0.1$ \\
\hline
\end{tabular}


against MRSA, respectively. Thus, both the carboxyl group and the exocyclic methylene group seem to be pivotal for the activity against these two clinically relevant strains.

\section{Antifungal Effects}

(-)-Polyalthic acid (1) was the most potent compound against Trichopyhton species dermatophytes in our previous study, with $\mathrm{IC}_{50}$ values of $6.8 \mu \mathrm{g} / \mathrm{ml}$ against $T$. rubrum and $4.3 \mu \mathrm{g} / \mathrm{ml}$ against $T$. mentagrophytes, respectively (Pfeifer Barbosa et al. 2019), while kaurenoic acid (2) only showed weak $(70.8 \mu \mathrm{g} / \mathrm{ml})$ to moderate effects $(15.5 \mu \mathrm{g} / \mathrm{ml})$ against these two strains (Table 3). Derivatisation of the two natural products this time revealed a different picture, leading to one equally potent (1b) and another still moderately active compound (1a) against $T$. rubrum, with $\mathrm{IC}_{50}$ values of $7.7 \mu \mathrm{g} / \mathrm{ml}$ for (-)-polyalthic acid methyl ester (1b) and $13.8 \mu \mathrm{g} / \mathrm{ml}$ for (-)-polyalthic acid $N, N$-diethylamide (1a), respectively. Notably, this effect was not observed against $T$. mentagrophytes, where the $\mathrm{IC}_{50}$ value was increased by the factor five. For kaurenoic acid (2), a similar picture was observed. Here, amidation (2a) and esterification (2b) yielded even more active compounds against $T$. rubrum, though on an overall higher level. Still, also against $T$. mentagrophytes the activity was drastically reduced by derivatisation of the carboxyl group. Of the remaining derivatives, only compound 1c showed activity, with an $\mathrm{IC}_{50}$ of $61.8 \mu \mathrm{g} / \mathrm{ml}$ against $T$. rubrum and $31.9 \mu \mathrm{g} / \mathrm{ml}$ against $T$. mentagrophytes.

The compounds were furthermore investigated for their inhibitory potential against two yeasts, Candida albicans and Cryptococcus neoformans. While the activity against C. albicans was negligible (Table S3), the results against C. neoformans revealed interesting findings for compounds 1, 1a and 1b. All three compounds showed similar $\mathrm{IC}_{50}$

Table 3 Antifungal effects of (-)-polyalthic acid (1), kaurenoic acid (2) and their derivatives. The $\mathrm{IC}_{50}$ values are in $\mu \mathrm{g} / \mathrm{ml}$. Positive controls were clotrimazole (Trichophyton rubrum and T. mentagrophytes) and amphotericin B (Cryptococcus neoformans)

\begin{tabular}{llll}
\hline & T. rubrum & T. mentagrophytes & C. neoformans \\
\hline Compound 1 & $6.8 \pm 0.1$ & $4.3 \pm 0.1$ & $11.2 \pm 0.3$ \\
Compound 1a & $13.8 \pm 3.0$ & $23.5 \pm 0.6$ & $9.5 \pm 0.4$ \\
Compound 1b & $7.7 \pm 0.6$ & $21.8 \pm 0.9$ & $11.0 \pm 4.9$ \\
Compound 1c & $61.8 \pm 1.3$ & $31.9 \pm 1.6$ & $>100$ \\
Compound 2 & $70.8 \pm 1.6$ & $15.5 \pm 0.7$ & $>100$ \\
Compound 2a & $51.5 \pm 7.0$ & $>100$ & $>100$ \\
Compound 2b & $53.0 \pm 6.2$ & $>100$ & $>100$ \\
Compound 2c & $>100$ & $>100$ & $>100$ \\
Compound 2d & $>100$ & $>100$ & $>100$ \\
Compound 2e & $>100$ & $>100$ & $>100$ \\
Positive control & $0.1 \pm 0.0$ & $0.1 \pm 0.0$ & $0.6 \pm 0.1$ \\
\hline
\end{tabular}

values, which were in the range of 9.5 to $11.2 \mu \mathrm{g} / \mathrm{ml}$ (Table 3). However, below this concentrations the activity of (-)-polyalthic acid (1) decreased rapidly in contrast to its two non-acidic derivatives (1a and $\mathbf{1 b}$ ) (Fig. 1).

\section{Conclusion}

(-)-Polyalthic acid (1) and kaurenoic acid (2) as well as their semi-synthetic derivatives (1a-1c and $\mathbf{2 a}-\mathbf{2 e})$ were investigated for their cytotoxic, antibacterial and antifungal properties, with different outcomes. While the previously discussed importance of an exocyclic methylene group for the cytotoxic effect was also apparent in our study, we furthermore found that both the exocyclic methylene and the carboxylic acid group were necessary for the effect against $E$. faecium and MRSA.

However, different results were obtained with regard to the antifungal activity of the two natural products and their derivatives. For T. mentagrophytes, the picture was somehow similar to the antibacterial assays, where the best antifungal activity was achieved when both an exocylic methylene group and a carboxylic acid were present. In contrast, the presence of an carboxyl group was not required for activity against C. neoformans and T. rubrum, as displayed by the similar $\mathrm{IC}_{50}$ values of $\mathbf{1}, \mathbf{1 a}$ and $\mathbf{1 b}$. However, the low $\mathrm{IC}_{50}$ value of compound 1c clearly shows that at least the methylene group is crucial for the effect.

Besides gaining further insights into differential biological activities and some SARs for copaiba diterpenoids, we present a novel semi-synthetic derivative of (-)-polyalthic acid. In the course of verifying the relative configuration of the new compound, we discovered ambiguities regarding the stereochemistry of polyalthic acid by two commonly used databases, which also led to incorrect chemical structures in several publications. We thus suggest to only use the terms (+)- and (-)-

\section{C. neoformans}

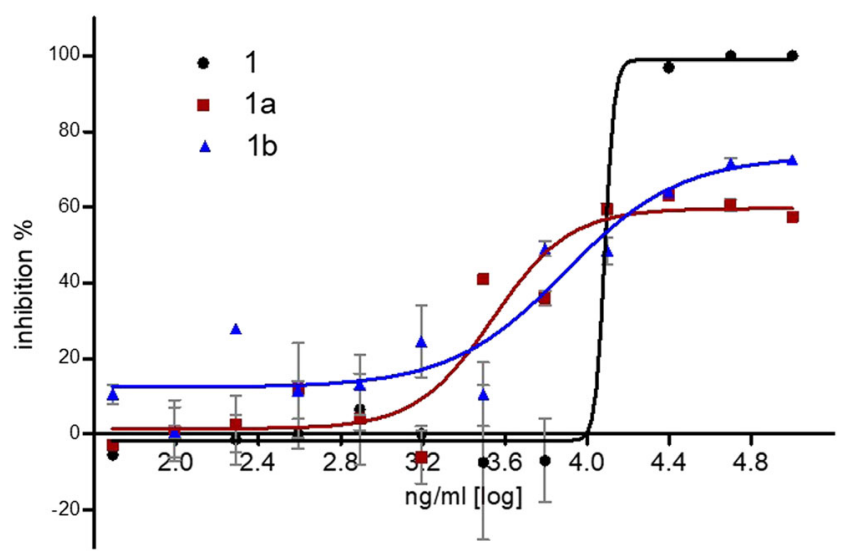

Fig. $1 \mathrm{IC}_{50}$ curves compounds $\mathbf{1}, \mathbf{1 a}$ and $\mathbf{1 b}$ against Cryptococcus neoformans 
polyalthic acid to differentiate between the two enantiomers in order to hopefully avoid such mistakes in future publications.

Acknowledgements The authors thank Jana Heumann for the measurements of the cell culture assays and Fengjie Li and Claudia Welsh for high-resolution MS and optical rotation measurements.

Author contribution SSC, AWS and UG conducted the experiments; DT supervised the bioactivity testing; AWS, UG and DT revised the manuscript; SSC designed the study and wrote the manuscript. All authors approved the final version of the manuscript.

Funding Information Open Access funding provided by Projekt DEAL.

\section{Compliance with Ethical Standards}

Conflict of Interest The authors declare that they have no conflict of interest.

Protection of Human and Animal Subjects The authors declare that no experiments were performed on humans or animals for this study.

Confidentiality of Data The authors declare that no patient data appear in this article.

Right to Privacy and Informed Consent The authors declare that no patient data appear in this article.

Open Access This article is licensed under a Creative Commons Attribution 4.0 International License, which permits use, sharing, adaptation, distribution and reproduction in any medium or format, as long as you give appropriate credit to the original author(s) and the source, provide a link to the Creative Commons licence, and indicate if changes were made. The images or other third party material in this article are included in the article's Creative Commons licence, unless indicated otherwise in a credit line to the material. If material is not included in the article's Creative Commons licence and your intended use is not permitted by statutory regulation or exceeds the permitted use, you will need to obtain permission directly from the copyright holder. To view a copy of this licence, visit http://creativecommons.org/licenses/by/4.0/.

\section{References}

Bardají DKR, da Silva JJM, Bianchi TC, Eugênio DD, de Oliveira PF, Leandro LF, Rogez HLG, Venezianni RCS, Ambrosio SR, Tavares DC, Bastos JK, Martins CHG (2016) Copaifera reticulata oleoresin: chemical characterization and antibacterial properties against oral pathogens. Anaerobe 40:18-27

Borges CHG, Cruz MG, Carneiro LJ, da Silva JJM, Bastos JK, Tavares DC, de Oiveira PF, Rodrigues V, Veneziani RCS, Parreira RLT, Caramori GF, Nagurniak GR, Magalhaes LG, Ambrosio SR (2016) Copaifera duckei oleoresin and its main nonvolatile terpenes: in vitro schistosomicidal properties. Chem Biodivers 13:1348-1356

Carneiro LJ, Bianchi TC, da Silva JJM, Oliveira LC, Borges CHG, Lemes DC, Bastos JK, Veneziani RCS, Ambrosio SR (2018) Development and validation of a rapid and reliable RP-HPLCPDA method for the quantification of six diterpenes in Copaifera duckei, Copaifera reticulata and Copaifera multijuga oleoresins. J Braz Chem Soc 29:729-737
Cavalcanti BC, Bezerra DP, Magalhaes HIF, Moraes MO, Lima MAS, Silveira ER, Camara CAG, Rao VS, Pessoa C, Costa-Lotufo LV (2009) Kauren-19-oic acid induces DNA damage followed by apoptosis in human leukemia cells. J Appl Toxicol 29:560-568

Chen CY, Chang FR, Cho CP, Wu YC (2000) Ent-Kaurane diterpenoids from Annona glabra. J Nat Prod 63:1000-1003

Chernov SV, Shul'ts EE, Shakirov MM, Tolstikov GA (2006) Synthetic transformations of higher terpenoids: XII.* Transformation of lambertianic acid into 14,16-epoxyabietane diterpenoids. Russ J Org Chem 42:36-41

Çiçek SS, Pfeifer Barbosa AL, Girreser U (2018) Quantification of diterpene acids in copaiba oleoresin by UHPLC-ELSD and heteronuclear two-dimensional qNMR. J Pharm Biomed Anal 160:126-134

Da Silva JJM, Crevelin EJ, Carneiro LJ, Rogez H, Veneziani RCS, Ambrosio SR, Moraes LAB, Bastos JK (2017) Development of a validated ultra-high-performance liquid chromatography tandem mass spectrometry method for determination of acid diterpenes in Copaifera oleoresins. J Chromatogr A 1515:81-90

Dauben WG, German VF (1965) Structure of lamertianic acid - a new diterpenic acid. Tetrahedron 22:679-683

Frøyen P (1994) A new general method for the preparation of carboxylic acid esters. Phosphorus Sulfur Silicon Relat Elem 91:145-151

Frøyen P (1995) The conversion of carboxylic acids into amides via NCS/ triphenylphosphine. Synth Commun 25:959-968

Gopinath KW, Govindachari GR, Parthasarathy PC, Viswanathan N (1961) Structure and stereochemistry of polyalthic acid, a new diterpene acid. Helv Chim Acta 44:1040-1049

Haeuser J, Ourisson G (1961) Stereochimie de l'acide daniellique. Tetrahedron 12:205-214

Izumi E, Ueda-Nakamura T, Veiga Junior VF, Pinto AC, Nakamura CV (2012) Terpenes from Copaifera demonstrated in vitro antiparasitic and synergistic activity. J Med Chem 55:2994-3001

Kubo I, Chaudhuri SK, Kubo Y, Sanchez Y, Ogura T, Saito T, Ishikawa H, Haraguchi H (1996) Cytotoxic and antioxidative sesquiterpenoids from Heterotheca inuloides. Planta Med 62:427430

Leandro, L.M., de Sousa Vargas, F., Barbosa, P.C.S., Neves, J.K.O., da Silva, J.A., da Veiga-Junior, V. F., 2012. Chemistry and biological activities of terpenoids from copaiba (Copaifera spp.) oleoresins. Molecules 17, 3866

Miyazawa M, Shimamura H, Nakamura S, Kameoka H (1995) Antimutagenic activity of (+)-polyalthic acid from Vitex rotundiforia. J Agric Food Chem 43:3012-3015

Mizuno CS, Souza AB, Tekwani BL, Ambrósio SR, Veneziani RCS (2015) Synthesis and biological evaluation of polyalthic acid derivatives for the treatment of neglected diseases. Bioorg Med Chem Lett 25:5529-5531

Narayanan CR, Venkatasubramanian NK (1965) Stereochemical studies by PMR spectroscopy - III, axial and equatorial acids. Tetrahedron Lett 41:3639-3646

Ohsaki A, Yan LT, Ito S, Edatsugi H, Iwata D, Komoda Y (1994) The isolation and in vivo potent antitumor activity of clerodane diterpenoid from the oleoresin of Brazilian medicinal plant Copaifera langsdorfii Desfon. Bioorg Med Chem Lett 4:2889-2892

Ohta K, Nawamaki T (1978) (+)-Polyalthic acid, a repellent against a sea snail Monodonta neritoides. Agric Biol Chem 42:1957-1958

Pfeifer Barbosa AL, Wenzel-Storjohann A, Barbosa JD, Zidorn C, Peifer C, Tasdemir D, Çiçek SS (2019) Antimicrobial and cytotoxic effects of the Copaifera reticulata oleoresin and its main diterpene acids. J Ethnopharmacol 233:94-100

Santos JO, Pereira GR, Brandao GC, Borgati TF, Arantes LM, de Paula RC, Soares LF, do Nascimento MFA, Ferreira MRC, Taranto AG, Varotti FP, de Oliveira AB (2016) Synthesis, in vitro antimalarial activity and in silico studies of hybrid kauranoid 1,2,3-triazoles 
derived from naturally occurring diterpenes. J Braz Chem Soc 27: $551-565$

Senedese JM, Rinaldi-Neto F, Furtado RA, Nicollela HD, de Souza LDR, Ribeiro AB, Ferreira LS, Magalhaes GM, Carlos IZ, da Silva JJM, Tavares DC, Bastos JK (2019) Chemopreventive role of Copaifera reticulata Ducke oleoresin in colon carcinogenesis. Biomed Pharmacother 111:331-337

Song JL, Yuan Y, Nie LH, Li BL, Qin XB, Li Y, Wu JW, Qiu SX (2018) A new ent-kaurane diterpene from the stems of Eurya chinensis R.Br. Nat Prod Res 32:182-188

Souza AB, Martins CHG, Souza MGM, Furtado NAJC, Heleno VCG, de Sousa JPB, Rocha EMP, Bastos JK, Cunha WR, Veneziani RCS, Ambrosio SR (2011) Antimicrobial activity of terpenoids from Copaifera langsdorffii Desf. Against cariogenic bacteria. Phytother Res 25:215-220
Tincusi BM, Jimenez IA, Bazzocchi IL, Moujir LM, Mamani ZA, Barroso JP, Ravelo AG, Hernandez BV (2002) Antimicrobial terpenoids from the oleoresin of the Peruvian medicinal plat Copaifera paupera. Planta Med 68:808-812

Vieira HS, Takahashi JA, de Oliveira AB, Chiari E, Boaventura MAD (2002) Novel derivatives of kaurenoic acid: preparation and evaluation of their trypanocidal activity. J Braz Chem Soc 13:151-157

Yaouba S, Valkonen A, Coghi P, Gao J, Guantai EM, Derese S, Wong VKW, Erdélyi M, Yenesew A (2018) Crystal structures and cytotoxicity of ent-kaurane-type diterpenoids from two Aspilia species. Molecules 23:3199

Yeo SK, Ali AY, Hayward OA, Turnham D, Jackson T, Bowen ID, Clarkson R (2016) Beta-bisabolene, a sesquiterpene from the essential oil extract of Opoponax (Commiphora guidottii), exhibits cytotoxicity in breast cancer cell lines. Phytother Res 30:418-425 